\title{
Off-diagonal wormhole and black hole deformations and dark energy in two measure/bi-metric modified gravity theories
}

\author{
Tamara Gheorghiu \\ Project IDEI, Alexandru Ioan Cuza University, UAIC \\ Al. Lapuşneanu str., nr. 14, UAIC - Corpus R, office 323; Iaşi, Romania, 700057 and \\ University of Medicine and Pharmacy "Gr. T. Popa" Iaşi \\ Faculty of Medicine, 16 University street, Iaşi, Romania, 700115 \\ E-mail: tamara.gheorghiu@yahoo.com
}

Olivia Vacaru

National College of Iaş i, 4 Arcu street, Iaşi, Romania, 700115

E-mail: olivia.vacaru@yahoo.com

Sergiu I. Vacaru

Rector's Department, Alexandru Ioan Cuza University, UAIC

Al. Lapuşneanu str., nr. 14, UAIC - Corpus R, office 323; Iaşi, Romania, 700057; and * M. Planck Inst. Physics (W. Heisenberg Inst.), Föhringer Ring 6, München D-80805, Germany; Leibniz Univ. Hannover, Instit. Theoretical Physics, Appelstrasse 2, Hannover 30167, Germany E-mails: sergiu.vacaru@uaic.ro; Sergiu.Vacaru@gmail.com

\begin{abstract}
We find general parameterizations for generic off-diagonal spacetime metrics and matter sources in general relativity (GR) and two measure/bi-metric modified gravity theories, MGTs, when the field equations decouple with respect to nonholonomic frames of reference. This allows us to construct various classes of exact solutions when the coefficients of the fundamental geometric/physical objects depend on all spacetime coordinates via corresponding classes of generating and integration functions and/or constants. Such (modified) spacetimes display Killing and non-Killing symmetries, describe nonlinear vacuum configurations and effective polarizations of cosmological and interaction constants. Certain examples of exact locally anisotropic wormhole and generic off-diagonal cosmological solutions are analysed for MGTs of massive and/or modified $\mathrm{f}(\mathrm{R}, \mathrm{T})$ gravity, two measure theory etc. We conclude that considering generic off-diagonal nonlinear parametric interactions in GR it is possible to mimic various effects in massive and/or modified gravity, or to distinguish certain classes of generic modified gravity solutions which cannot be encoded in GR.
\end{abstract}

Keywords: Modified and massive gravity; two measure theories; effective Einstein gravity; off-diagonal wormhole, black hole and cosmological solutions; dark energy.

\section{Introduction}

In a series of works $^{1-5}$, we developed a geometric approach (the anholonomic frame deformation method, AFDM) for constructing generic off-diagonal exact solutions in modified gravity theories, MGTs, and general relativity, GR. Such a geometric techniques involve re-formulation of physical models in certain classes of nonholonomic variables (nonintegrable frames with distortion of connections) when the gravitational and matter field equations decouple as certain systems of

*DAAD fellowship affiliations for two host institutions 
nonlinear partial differential equations, PDEs, which can be integrated in almost general forms.

Following geometric methods, we can integrate (effective) Einstein equations for generalized metrics, connections and sources as nonholonomic nonlinear systems of PDEs, when the solutions are with generic off-diagonal metrics depending on all spacetime coordinates via generating and integration functions and various integration constants. Prescribing corresponding classes of generating and integration functions with generalized symmetries and respective boundary/initial conditions, we can generate various classes of exact solutions in MGTs modelling different, or equivalent, types of nonlinear parametric interactions. Such classes of "generalized solutions" are very different from the well known and physically important black hole, wormhole and/or cosmological solutions with particular type (for instance, spherical, or cylindrical) symmetry determined by integration constants. Mathematically, the new classes of solutions can be encoded and parameterized as solitonic wave hierarchies ${ }^{6}$. For physicists, it is very important to study how such generalized solutions are related, for instance, to nonholonomic deformations of the Kerr and wormhole metrics ${ }^{1,3}$ and to homogeneous and inhomogeneous cosmological solutions in various $\mathrm{MGTs}^{2,5}$. Another important issue is that how certain classes of (off) diagonal solutions may mimic similar physical effects in theories of different types when, for instance, dark matter and dark energy cosmological observation data can be explained by parametric nonlinear effects in some (effective) GR models with non-minimal coupling and nontrivial vacuum structure ${ }^{5,7}$.

In the present paper, we elaborate on possible equivalent modelling in MGTs and GR when generic off-diagonals solutions of effective Einstein equations are found for two measure theories, TMTs, and corresponding bi-metric gravity models. The corresponding actions are

$$
\begin{aligned}
\mathcal{S}= & \left({ }^{P l} M\right)^{2} \int d^{4} u \sqrt{|\widehat{\mathbf{g}}|}[\widehat{R}+\widehat{\mathcal{L}}] \\
= & { }^{\Phi} \mathcal{S}+{ }^{m} \mathcal{S}=\int d^{4} u{ }^{1} \Phi(\mathbf{A})\left[\widehat{R}+{ }^{1} L\right] \\
& +\int d^{4} u^{2} \Phi(\mathbf{B})\left[{ }^{2} L+\epsilon \mathbf{f}(\widetilde{\mathbf{R}})+\left(\sqrt{|\mathbf{g}|}^{-1} \Phi(\mathbf{H})\right]+\int d^{4} u \sqrt{|\widehat{\mathbf{g}}|}^{m} \mathcal{L}\right. \\
= & { }^{F, \mu} \mathcal{S}+{ }^{m} \mathcal{S}=\left({ }^{P l} M\right)^{2} \int d^{4} u\left[\sqrt{|\widehat{\mathbf{g}}|}^{F, \mu} \mathcal{L}+\sqrt{|\widehat{\mathbf{g}}|}^{m} \mathcal{L}\right],
\end{aligned}
$$

where $|\widehat{\mathrm{g}}|=\operatorname{det}\left|\widehat{\mathrm{g}}_{\alpha \beta}\right|$ is for a metric, $\widehat{\mathrm{g}}_{\alpha \beta}$, constructed effectively by a conformal transform of a TMT reference one, $\mathbf{g}_{\alpha \beta}$ (see below); ${ }^{\Phi} L$ defines a class of theories with two independent non-Riemannian volume-forms ${ }^{1} \Phi(A)$ and ${ }^{2} \Phi(B)$ with a more general functional for modification, of type $\epsilon \mathbf{f}(\widetilde{\mathbf{R}})$, than $\epsilon R^{2}$ if the generalized connection $\widehat{\mathbf{D}}$ transforms into the Levi-Civita one $\nabla$; the Lagrange density functional ${ }^{f, \mu} \mathcal{L}=\mathbf{F}(\widetilde{\mathbf{R}})$ is determined similarly to a modified massive gravity theory by a mass-deformed scalar curvature

$$
\tilde{\mathbf{R}}:=\widehat{\mathbf{R}}+2 \mu^{2}(3-\operatorname{tr} \sqrt{\mathbf{q} / \mathbf{g}}-\operatorname{det} \sqrt{\mathbf{q} / \mathbf{g}}),
$$


where $\mu$ is the graviton's mass and $\mathbf{q}=\left\{\mathbf{q}_{\alpha \beta}\right\}$ is the so-called non-dynamical reference metric; ${ }^{m} \mathcal{L}$ is the Lagrangian for matter fields; see corresponding details in references ${ }^{1-5}$.

A variational $\mathrm{N}$-adapted calculus on form fields $\mathbf{A}, \mathbf{B}, \mathbf{H}$ of actions (1) and metric $\mathrm{g}$ results in effective gravitational field equations

$$
\widehat{\mathbf{R}}_{\mu \nu}\left[\widehat{\mathbf{g}}_{\alpha \beta}\right]={ }^{e f} \widehat{\boldsymbol{\Upsilon}}_{\mu \nu}+{ }^{F, \mu} \widehat{\boldsymbol{\Upsilon}}_{\mu \nu},
$$

where ${ }^{F, \mu} \widehat{\Upsilon}_{\mu \nu}$ is determined by variation of ${ }^{F, \mu} \mathcal{S}+{ }^{m} \mathcal{S}$ and

$$
{ }^{e f} \widehat{\Upsilon}_{\beta \gamma}:=\varkappa\left({ }^{e f} \widehat{\mathbf{T}}_{\alpha \beta}-\frac{1}{2} \widehat{\mathbf{g}}_{\alpha \beta}{ }^{e f} \widehat{T}\right)
$$

is computed for $\mathbf{g}_{\alpha \beta} \rightarrow \widehat{\mathbf{g}}_{\alpha \beta}$ and $\widehat{\mathcal{L}} \rightarrow{ }^{e f} \mathcal{L}$, where $\widehat{\mathbf{g}}_{\alpha \beta}=\Theta \mathbf{g}_{\alpha \beta}$, for

$$
\begin{aligned}
& \Theta={ }^{1} \chi-{ }^{2} \chi \epsilon{ }^{1} \mathbf{f}\left({ }^{1} L+{ }^{1} M, \mu\right) \\
& \Theta{ }^{e f} L={ }^{1} L+{ }^{1} M+{ }^{2} \chi\left[{ }^{2} L+{ }^{1} M+\epsilon{ }^{1} \mathbf{f}\left({ }^{1} L+{ }^{1} M, \mu\right)\right] / \Theta,
\end{aligned}
$$

when the conformal factor $\Theta$ for the Weyl re-scaling of d-metric is induced by the nonlinear functional in the action

$$
{ }^{1} \mathbf{f}\left({ }^{1} L+{ }^{1} M, \mu\right)=\left.\frac{d \mathbf{f}(\widehat{\mathbf{R}}, \mu)}{d \widehat{\mathbf{R}}}\right|_{\widehat{\mathbf{R}}={ }^{1} L+{ }^{1} M}
$$

and the two measure functionals ${ }^{1} \chi={ }^{1} \Phi(\mathbf{A}) / \sqrt{\left|\widehat{\mathbf{g}}_{\mu \nu}\right|}$ and ${ }^{2} \chi={ }^{2} \Phi(\mathbf{B}) / \sqrt{\left|\widehat{\mathbf{g}}_{\mu \nu}\right|}$.

We found three important classes of solutions for (2) for generic off-diagonal deformations of physically important solutions in GR.

\section{Ellipsoid Kerr-de Sitter configurations}

We construct a subclass of solutions with rotoid configurations for generating functions ${ }^{1} \tilde{\Phi}=e^{\varpi}\left[\tilde{\Lambda} / 2^{\mu} \tilde{\Lambda}+\underline{\zeta} \sin \left(\omega_{0} \varphi+\varphi_{0}\right)\right]$, for $\tilde{\Phi}=e^{\varpi}$, (see details in $\left.{ }^{3}\right)$,

$$
\begin{aligned}
d s^{2}= & e^{\psi\left(x^{k^{\prime}}\right)}\left(1+\varepsilon \chi\left(x^{k^{\prime}}\right)\right)\left[\left(d x^{1^{\prime}}\right)^{2}+\left(d x^{2^{\prime}}\right)^{2}\right]-\frac{e^{2 \varpi}}{4 \mid \mu \tilde{\Lambda}} \bar{A}\left[1+2 \varepsilon \underline{\zeta} \sin \left(\omega_{0} \varphi+\varphi_{0}\right)\right] \\
& \times\left[d y^{3^{\prime}}+\left(\partial_{k^{\prime}}{ }^{\eta} n\left(x^{i^{\prime}}\right)-\partial_{k^{\prime}}\left(\widehat{y}^{3^{\prime}}+\varphi \bar{B} / \bar{A}\right)\right) d x^{k^{\prime}}\right]^{2} \\
& +\frac{\left(\varpi^{*}\right)^{2}}{\mu \tilde{\Lambda}}\left(\bar{C}-\bar{B}^{2} / \bar{A}\right)\left[1+\varepsilon\left(\partial_{4} \varpi \tilde{\Lambda} / \tilde{\lambda}+2 \partial_{4} \varpi \underline{\zeta} \sin \left(\omega_{0} \varphi+\varphi_{0}\right)\right.\right. \\
& \left.\left.+2 \omega_{0} \underline{\zeta} \cos \left(\omega_{0} \varphi+\varphi_{0}\right)\right)\right]\left[d \varphi+\left(\partial_{i^{\prime}} \tilde{A}+\varepsilon \partial_{i^{\prime}}{ }^{1} \check{A}\right) d x^{i^{\prime}}\right]^{2} .
\end{aligned}
$$

Such metrics have a Killing symmetry in $\partial / \partial y^{3}$ and are completely defined by a generating function $\varpi\left(x^{k^{\prime}}, \varphi\right)$ and the sources $\mu \tilde{\Lambda}=\mu_{g}^{2} \lambda$ (for massive gravity terms) and $\widetilde{\Lambda}$. They describe $\varepsilon$-deformations of Kerr-de Sitter black holes into ellipsoid configurations with effective (polarized) cosmological constants determined by the constants in massive gravity and $f$-modifications. If the zero torsion conditions are satisfied, such metrics can be modelled in GR. 


\section{Nonholonomically deformed wormhole configurations ${ }^{1}$}

Such metrics are parameterized

$$
\begin{aligned}
\mathbf{d s}^{2}= & e^{\tilde{\psi}(\widetilde{\xi}, \theta)}\left(d \widetilde{\xi}^{2}+d \vartheta^{2}\right) \\
& +\frac{\left[\partial_{\varphi} \widetilde{\varpi}\right]^{2}}{\mu_{g}^{2} \lambda}\left(1+\varepsilon \frac{\partial_{\varphi}\left[\bar{\chi}_{4} \widetilde{\varpi}\right]}{\partial_{\varphi} \widetilde{\varpi}}\right){ }^{0} \bar{h}_{3}\left[d \varphi+\partial_{\widetilde{\xi}}\left({ }^{\eta} \widetilde{A}+\varepsilon \bar{A}\right) d \widetilde{\xi}+\partial_{\vartheta}\left({ }^{\eta} \widetilde{A}+\varepsilon \bar{A}\right) d \vartheta\right]^{2} \\
& -\frac{e^{2 \widetilde{\varpi}}}{4 \mu_{g}^{2}|\lambda|}\left[1+\varepsilon \bar{\chi}_{4}(\widetilde{\xi}, \varphi)\right] e^{2 B(\widetilde{\xi})}\left[d t+\partial_{\widetilde{\xi}}\left({ }^{\eta} n+\varepsilon \bar{n}\right) d \widetilde{\xi}+\partial_{\vartheta}\left({ }^{\eta} n+\varepsilon \bar{n}\right) d \vartheta\right]^{2} .
\end{aligned}
$$

If the generating functions $\widetilde{\varpi}$ and effective source $\lambda$ in massive gravity are such way chosen that effective polarization functions can be approximated $\widetilde{\eta}_{a} \simeq 1$ and $\eta \widetilde{A}$ and ${ }^{\eta} n$ are "almost constant", with respect to certain systems of radial coordinates, the metric (3) mimic small rotoid wormhole like configurations with off-diagonal terms and $f$-modifications of the diagonal coefficients. It is possible to chose such integration functions and constants that this class of stationary solutions define wormhole like metrics depending generically on three space coordinates with self-consistent "imbedding" in an effective massive gravity background, for ${ }^{0} \bar{h}_{3}=r^{2}(\widetilde{\xi}) \sin ^{2} \theta(\widetilde{\xi}, \vartheta)$, where $\widetilde{\xi}=\int d r / \sqrt{|1-b(r) / r|}$ and $B(\widetilde{\xi})$ are determined by a wormhole metric.

\section{Soitonic waves for inhomogeneous cosmological solutions}

For simplicity, we can consider solutions by a nonlinear radial (solitonic, with left $s$-label) generating function $\Phi={ }^{s} \check{\Phi}(r, t)=4 \arctan e^{q \sigma(r-v t)+q_{0}}$ and construct a metric

$$
\begin{aligned}
& \mathbf{d s}^{2}=e^{\psi(r, \theta)}\left(d r^{2}+d \theta^{2}\right)+\frac{{ }^{s} \check{\Phi}^{2}}{4\left({ }^{m} \Upsilon+{ }^{\alpha} \Upsilon\right)} \stackrel{\circ}{h}_{3}(r, \theta) d \varphi^{2} \\
& -\frac{\left(\partial_{t}{ }^{s} \check{\Phi}\right)^{2}}{\left({ }^{m} \Upsilon+{ }^{\alpha} \Upsilon\right)^{s} \check{\Phi}^{2}} \stackrel{\circ}{h}_{4}(r, t)\left[d t+\left(\partial_{r} \widetilde{A}\right) d r\right]^{2} \text {. }
\end{aligned}
$$

In this metric, for simplicity, we fixed $n(r, \theta)=0$ and consider that $\widetilde{A}(r, t)$ is defined as a solution of ${ }^{s} \check{\Phi}^{\bullet} /{ }^{s} \check{\Phi}^{*}=\partial_{r} \widetilde{A}$ and $\stackrel{h}{h}_{a}$ are given by a homogeneous cosmology model data. The generating function is just a 1-soliton solution of the sine-Gordon equation

$$
{ }^{s} \check{\Phi}^{* *}-{ }^{s} \check{\Phi}^{\bullet \bullet}+\sin ^{s} \check{\Phi}=0 .
$$

For any class of small polarizations with $\eta_{a} \sim 1$ ), we can consider that the source $\left({ }^{m} \Upsilon+{ }^{\alpha} \Upsilon\right)$ is polarized by ${ }^{s} \check{\Phi}^{-2}$ when $h_{3} \sim \stackrel{\circ}{h}_{3}$ and $h_{4} \sim \stackrel{\circ}{h}_{4}\left({ }^{s} \check{\Phi}^{*}\right)^{2} /{ }^{s} \check{\Phi}^{4}$ with an off-diagonal term $\partial_{r} \widetilde{A}$ resulting in a stationary solitonic universe. If we consider that $\left(\partial_{\widehat{R}} \widehat{f}\right)^{-1}={ }^{s} \check{\Phi}^{-2}$, we can model $\widehat{f}$-interactions via off-diagonal interactions and "gravitational polarizations".

In absence of matter, ${ }^{m} \Upsilon=0$, the off-diagonal cosmology is completely determined by ${ }^{\alpha} \Upsilon$ when ${ }^{s} \check{\Phi}$ transforms $\stackrel{\mu}{\mu}$ into an anisotropically polarized/variable mass of solitonic waves. Such configurations can be modelled if ${ }^{m} \Upsilon \ll{ }^{\alpha} \Upsilon$. If 
${ }^{m} \Upsilon \gg{ }^{\alpha} \Upsilon$, we generate cosmological models determined by distribution of matter fields when contributions from massive gravity are with small anisotropic polarization, see details in ${ }^{2,5,7}$.

\section{Acknowledgments}

$\mathrm{SV}$ is supported by a travel grant from MG14 and reports certain research related to his basic activity at UAIC, a DAAD fellowship and the Program IDEI, PN-II-IDPCE-2011-3-0256. He is grateful for DAAD hosting to D. Lüst and O. Lechtenfeld. TG communicates certain research results following the Program IDEI, PN-II-IDPCE-2011-3-0256. OV participates as a young researcher.

\section{References}

1. S. Vacaru, Eur. Phys. J. C 74, 2781 (2014); arXiv: 1403.1815.

2. S. Vacaru, Eur. Phys. J. C 74, 3132 (2014); arXiv: 1401.2882.

3. T. Gheorghiu, O. Vacaru, S. Vacaru, Eur. Phys. J. C 74, 3152 (2014); arXiv: 1312.4844.

4. T. Gheorghiu, O. Vacaru, S. Vacaru, Class. Quant. Gr. 32, 065004 (2015); arXiv: 1311.6365.

5. S. Vacaru, Eur. Phys. J. C 75, 176 (2015); arXiv: 1504.04346.

6. S. Vacaru, Mod. Phys. Lett. A 30, nr. 19, 1550090 (2015); arXiv: 1308.6180.

7. E. Elizalde, S. Vacaru, Gen. Relat. Grav. 47, 64 (2015). 